\title{
EFFETS D'UN HYDROCARBURE AROMATIQUE POLYCYCLIQUE ET D'UN PCB SUR LES ACTIVITÉS DE MÉTABOLISATION DES XÉNOBIOTIQUES DE LA TRUITE ARC-EN-CIEL (O. MYKISS).
}

\author{
J.P. CRAVEDI, E. PERDU-DURAND et E. POUPIN
}

Laboratoire des Xénobiotiques, INRA, BP 3, 31931 Toulouse Cedex, France.

\section{RÉSUMÉ}

Des truites arc-en-ciel immatures ont été soumises à des conditions statiques d'exposition à 10 et $100 \mu \mathrm{g} / \mathrm{l}$ de 3,3', 4,4'-tétrachlorobiphényle (TCB) ou de benzo(a)pyrène (BaP) dissous dans l'acétone, pendant $72 \mathrm{~h}$, puis transférées en eau courante non contaminée. Parallèlement un groupe de poissons a servi de lot témoin. Une partie des poissons a été sacrifiée en fin de période d'exposition, l'autre partie faisant l'objet d'un abattage après 2 semaines en eau propre. Le foie, les rosettes olfactives, les branchies et le rein ont été prélevés sur les poissons et, des microsomes et cytosols ont été préparés pour chacun des tissus par ultracentrifugation dans un tampon approprié. Les activités éthoxyrésorufine-o-dééthylase (EROD), glucuronosyl transférase (UDPGT) et glutathion-Stransférase (GST) ont été mesurées.

Les résultats de l'exposition au BaP montrent qu'aux deux concentrations testées, l'activité EROD a été significativement augmentée par rapport au lot témoin, pour le foie ( $x 11$ à 33 fois) et la branchie ( $x 5$ à 7 fois). Seule la dose la plus élevée a permis d'augmenter l'activité de cette enzyme dans le rein et la rosette olfactive. A la fin de la période de dépuration, il n'est observé aucune différence significative entre les lots traités et témoins. Les transférases apparaissent peu sensibles au B(a)P.

Après $72 \mathrm{~h}$ d'exposition au TCB, l'activité EROD du foie, des branchies, des rosettes et du rein est très significativement augmentée ( 5 à 50 fois selon le tissu) par rapport au témoin, aux deux concentrations testées. Cette induction est encore plus nette après la période de 2 semaines en eau propre. L'activité UDPGT hépatique est doublée en fin de traitement pour les deux doses et se maintient à ce niveau au cours de la période de dépuration. Les GST sont légèrement induites dans le foie après les 15 jours de dépuration.

Ce travail confirme la grande sensibilité de l'activité EROD des truites arc-en-ciel aux hydrocarbures aromatiques polycycliques et aux $\mathrm{PCB}$ et souligne les possibilités et les limites d'utilisation de cette activité en tant que biomarqueur d'exposition à ces polluants.

Mots-clés : enzymes de biotransformation, biomarqueur, EROD, cytochrome P450, $\mathrm{PCB}$, hydrocarbures aromatiques polycycliques, polluants, truite arc-en-ciel.

\author{
EFFECTS OF A POLYCYCLIC AROMATIC HYDROCARBON AND A PCB \\ ON XENOBIOTIC METABOLIZING ACTIVITIES IN RAINBOW TROUT (O. MYKISS).
}

\section{ABSTRACT}

Immature rainbow trout were exposed under static conditions to 3,3', 4,4'-tetrachlorobiphenyl (TCB) or benzo(a)pyrene (BaP) concentrations of 10 and 
$100 \mu \mathrm{g} / \mathrm{l}$, using acetone as carrier, for $72 \mathrm{~h}$, followed by depuration in clean water for 2 weeks. A group of control fish was exposed for $72 \mathrm{~h}$ to acetone only. Liver, gills, olfactory epithelium and kidney were sampled in control and exposed fish at the end of the exposure and depuration periods. Microsomes and cytosols were prepared from each tissue by differential centrifugation in an appropriate buffer. Ethoxyresorufin-o-deethylase (EROD), glucuronyl transferase (UDPGT) and glutathion-S-transferase (GST) were the activities measured in each tissue.

The results of the exposure study showed that at both $\mathrm{BaP}$ concentrations, EROD activity was significantly increased compared to controls in liver ( 11 to 33 fold) and in gill (5 to 7 fold). In kidney and olfactive organ, EROD activity was significantly increased only with the $100 \mathrm{ppb}$ exposure. At the end of the depuration period, no difference was observed between control and treated fish. Transferases remained almost unaffected by this hydrocarbon.

The exposure of rainbow trout to both TCB concentrations resulted in a 5 - 50 fold increase of EROD activity in liver, gill, olfactive epithelium and kidney. This induction was even more important at the end of the depuration period. Liver UDPGT activity in TCB exposed fish was 2 fold higher than in controls and remained at this level during the depuration period. A slight increase of hepatic GST activity was observed after the 2 weeks depuration period.

This work confirms the sensitivity of EROD activity to polycyclic aromatic hydrocarbons and PCBs and the possibilities and limits of this system as a pollution biomarker are discussed.

Key-words : biotransformation enzymes, biomarker, EROD, cytochrome P450, PCB, polycyclic aromatic hydrocarbons, pollutants, rainbow trout.

\section{INTRODUCTION}

En matière d'évaluation et de prévention des risques, le besoin d'indicateurs sensibles permettant le contrôle des écosystèmes aquatiques va croissant. Dans ce but, l'étude des variations de paramètres biochimiques, et en particulier des activités de biotransformation sous l'impact de contaminants, est séduisante. Cette approche est d'autant plus intéressante qu'il n'est en général pas possible, en matière de surveillance du milieu, d'identifier et d'analyser en permanence la totalité des polluants chimiques présents à l'état de traces. Parmi les systèmes proposés par les écotoxicologues comme indicateur possible de pollution figurent les monooxygénases à cytochrome P450 (LINDSTRÖMSEPPÄ et al., 1985 ; PAYNE et al., 1987 ; VINDIMIAN et GARRIC, 1989 ; NARBONNE et al., 1991) et à un degré moindre, les transférases (ANDERSSON et al., 1985 ; JEDAMSKIGRYMLAS et al., 1995). De nombreuses publications démontrent que des polluants majeurs de l'environnement tels que les hydrocarbures aromatiques polycycliques (HAP) ou les polychlorobiphényles (PCB) sont des inducteurs puissants du CYP1A, un des isozymes du cytochrome P450 (MONOD et al., 1988 ; GOKSØYR et FÖRLIN, 1992 ; HAASCH et al., 1993). Cependant, la plupart de ces travaux ne considérant que les enzymes hépatiques mettent en oeuvre des doses importantes de polluants ou utilisent des voies d'exposition telles que l'injection intrapéritonéale très éloignées de la situation de terrain.

L'objectif de notre étude a été de mesurer chez la truite arc-en-ciel l'effet de deux contaminants, le 3,3', 4,4'-tétrachlorobiphényle (TCB) et le benzo(a)pyrène (BaP), utilisés respectivement comme modèle de $\mathrm{PCB}$ et de HAP sur les enzymes de biotransformation situées dans le foie, la branchie, le rein et la rosette olfactive. Les concentrations de ces polluants dans les milieux naturels contaminés peuvent dépasser $10 \mathrm{ppb}$ (JONES et al., 1980) mais se situent généralement entre 0,1 et $0,5 \mathrm{ppb}$ (BOULOUBASSI et SALIOT, 1993; CHEVREUIL et al., 1995). Les activités de l'éthoxyrésorufine-o-dééthylase (EROD), enzyme dépendant du CYP1A, mais également celles de l'UDP-glucuronosyl transférase et 
la glutathion-transférase, deux enzymes de conjugaison, ont été déterminées après $72 \mathrm{~h}$ d'exposition par balnéation à deux concentrations (10 ppb et $100 \mathrm{ppb}$ ) de TCB ou de BaP. Nous avons également mesuré l'évolution de ces activités enzymatiques après le passage en eau non contaminée des poissons exposés au BaP ou au TCB.

\section{MATÉRIEL ET MÉTHODES}

Le TCB, le BaP, l'éthoxyrésorufine, le chlorodinitrobenzène, le NADPH et le 4-nitro[U-14 $\mathrm{C}]$-phénol proviennent de chez Sigma-chimie. Tous les autres produits chimiques utilisés dans cette expérimentation sont de qualité analytique.

Les truites arc-en-ciel (Oncorhynchus mykiss) d'un poids moyen de $180 \mathrm{~g}$ proviennent d'une pisciculture de I'INRA à Donzacq (Landes) et sont acclimatées pendant au moins 2 semaines avant l'expérimentation à l'eau de ville préalablement filtrée sur charbon actif. Elles sont réparties en 6 lots de 30 individus dans des bacs de 150 litres. La température de l'eau est de $12 \pm 1^{\circ} \mathrm{C}$ et la photopériode réglée à $12 \mathrm{~h}$ de jour/12 $\mathrm{h}$ de nuit. Les poissons reçoivent ad libitum, et une fois par jour, sauf pendant la période d'exposition, de l'aliment sous forme de granulés (Trouvit, Vervins). Au début de l'expérimentation, l'alimentation en eau des bacs est coupée et un bullage d'oxygène est installé. Les truites sont alors soumises pendant $72 \mathrm{~h}$ à une exposition statique au TCB et au BaP à deux concentrations : $10 \mu \mathrm{g} / \mathrm{l}$ et $100 \mu \mathrm{g} / \mathrm{l}$. Chaque dose est dissoute dans $500 \mu \mathrm{l}$ d'acétone avant son introduction dans le bac. Les deux lots restants constituent les lots témoins et sont traités par l'acétone uniquement. A la fin de la période d'exposition, 15 truites sont prélevées dans chaque lot et sont abattues. Le foie, les branchies, la rosette olfactive et le rein postérieur sont prélevés pour la préparation des fractions subcellulaires. Les truites restantes sont transférées dans des bacs non contaminés, alimentés en eau de ville déchlorée. Après 2 semaines en eau propre (période de dépuration), les poissons sont sacrifiés et les mêmes tissus que précédemment sont prélevés pour la préparation des fractions subcellulaires.

Les microsomes et les cytosols des différents tissus ont été préparés selon la technique décrite par MONOD et al. (1994). L'activité EROD a été mesurée à $20^{\circ} \mathrm{C}$ à partir des microsomes de chaque tissu (MONOD et al., 1994). L'activité UDPGT a été déterminée à partir du 4-nitro[U- $\left.{ }^{14} \mathrm{C}\right]$-phénol comme substrat selon la méthode de TUKEY et al. (1978). L'activité de la GST cytosolique a été suivie en spectrophotométrie (HABIG et JAKOBY, 1981). Les teneurs en protéines ont été mesurées selon la technique de LOWRY et al. (1951) avec l'albumine sérique bovine comme référence.

Les moyennes des activités de biotransformations des lots traités ont été comparées à celles des lots témoins par un test $t$ de Student.

\section{RÉSULTATS}

Les résultats concernant le $\mathrm{B}(\mathrm{a}) \mathrm{P}$ sont présentés dans le Tableau I. Ils montrent qu'à $100 \mu \mathrm{g} / \mathrm{l}$, l'activité EROD est significativement augmentée dans tous les tissus. Le niveau d'activité est multiplié par 33 pour le foie et par 5 à 7 dans les autres tissus. A faible dose $(10 \mu \mathrm{g} / \mathrm{l})$, seules les activités hépatiques et branchiales augmentent de façon significative ( $\times 11$ et $\times 5$ respectivement). A forte dose $(100 \mu \mathrm{g} / \mathrm{l})$, tous les tissus voient leurs niveaux d'activité EROD augmenter significativement. Après deux semaines de dépuration, on observe un retour aux valeurs de base des niveaux d'activité. Ce retour à la situation initiale permet de constater que l'absence de groupes témoins à $72 \mathrm{~h}$ et en fin de période de dépuration n'a pas d'influence sur l'interprétation des données. Les activités de conjugaison apparaissent peu ou pas sensibles au B(a)P. Seule l'UDPGT branchiale est augmentée lorsque les truites sont exposées à $100 \mu \mathrm{g} / \mathrm{l}$ de $\mathrm{B}(\mathrm{a}) \mathrm{P}$. 


\section{Tableau I}

Effet d'une exposition au BaP sur l'activité des enzymes du métabolisme des xénobiotiques dans différents tissus de truite. Les mesures ont été effectuées après une balnéation de $72 \mathrm{~h}$, puis un transfert en eau non contaminée de 2 semaines et sont exprimées en pmoles $/ \mathrm{min} / \mathrm{mg}$ de protéines (EROD) ou $\mathrm{nmoles} / \mathrm{min} / \mathrm{mg}$ de protéines (UDPGT, GST). Les valeurs sont des moyennes I écarts types ; $\mathbf{n}=\mathbf{3}$ groupes de 5 poissons ; ${ }^{\star \star \star}$ : significativement différentes du lot témoin (respectivement $p<0,05$ et $p<0,01$ ).

\section{Table I}

Xenobiotic metabolizing enzyme activities in various tissues (liver, gill, olfactive epithelium and kidney) of trout exposed to BaP. Measurements were carried out after $72 \mathrm{~h}$ waterborne exposure followed by a 2 weeks transfert in uncontaminated water. Values are expressed as $\mathrm{pmol} / \mathrm{min} / \mathrm{mg}$ protein (EROD) or $\mathrm{nmol} / \mathrm{min} / \mathrm{mg}$ protein (UDPGT, GST) and represent means \pm SD, $n=3$ groups of 5 fishes ; ${ }^{\star \star}$ : significantly different from controls ( $p<0.05$ and $p<0.01$, respectively).

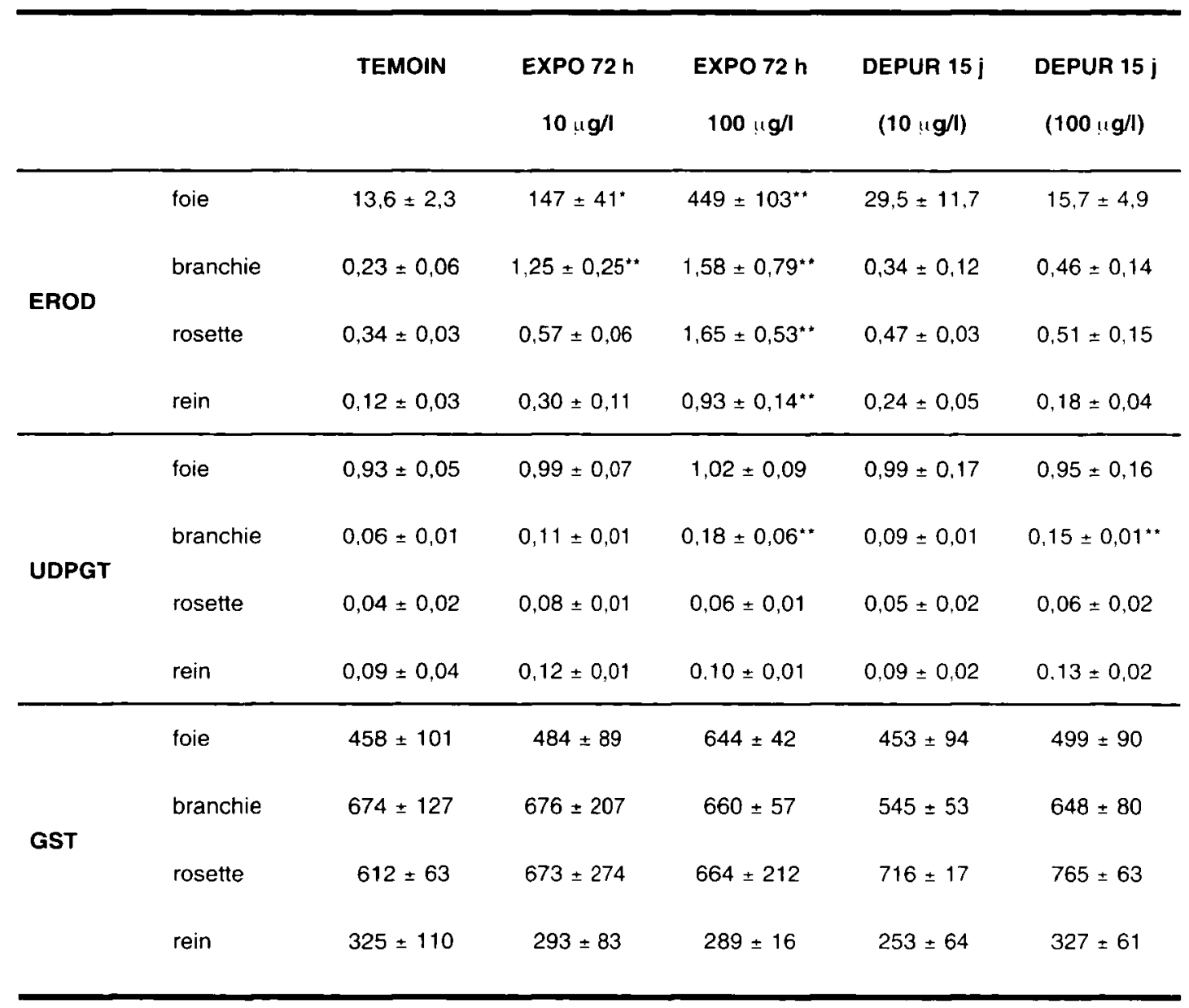

Après $72 \mathrm{~h}$ d'exposition à $10 \mu \mathrm{g} / \mathrm{l}$ de TCB, l'activité EROD est multipliée par 20 environ dans le foie, par 25 dans la branchie et par 55 dans le rein (Tableau II). Cette augmentation est sensiblement du même ordre lorsque la concentration en TCB dans l'eau est de $100 \mu \mathrm{g} / \mathrm{l}$. Contrairement au B(a)P, ces niveaux d'activité restent élevés, voire continuent à augmenter après deux semaines de dépuration. Ainsi l'activité EROD branchiale à la fin 
de l'expérimentation était, elle, pour le lot préalablement contaminé par $10 \mu \mathrm{g} / \mathrm{lde} \mathrm{TCB}$, 126 fois supérieure à celle mesurée dans le lot témoin. De façon plus modérée, l'activité UDPGT hépatique et branchiale est, elle aussi, augmentée par la contamination par le TCB. Comme pour l'activité EROD, ces niveaux restent élevés après l'arrêt de la contamination. Les GST sont légèrement induites dans le foie après les 15 jours de dépuration.

\section{Tableau II}

Effet d'une exposition au TCB sur l'activité des enzymes du métabolisme des xénobiotiques dans différents tissus de truite. Les mesures ont été effectuées après une balnéation de $72 \mathrm{~h}$, puis un transfert en eau non contaminée de 2 semaines et sont exprimées en pmoles/min/mg de protéines (EROD) ou en $\mathrm{nmoles} / \mathrm{min} / \mathrm{mg}$ de protéines (UDPGT, GST). Les valeurs sont des moyennes \pm écarts types $; n=3$ groupes de 5 poissons ; ${ }^{*},{ }^{\star *}$ : significativement différentes du lot témoin (respectivement $p<0,05$ et $p<0,01$ ).

Table II

Xenobiotic metabolizing enzyme activities in various tissues (liver, gill, olfactive epithelium and kidney) of trout exposed to TCB. Measurements were carried out after $72 \mathrm{~h}$ waterborne exposure followed by a 2 weeks transfert in uncontaminated water. Values are expressed as $\mathrm{pmol} / \mathrm{min} / \mathrm{mg}$ protein (EROD) or $\mathrm{nmol} / \mathrm{min} / \mathrm{mg}$ protein (UDPGT, GST) and represent means \pm SD, $n=3$ groups of 5 fishes ; ${ }^{\star},{ }^{\star}$ : significantly different from controls ( $p<0.05$ and $p<0.01$, respectively).

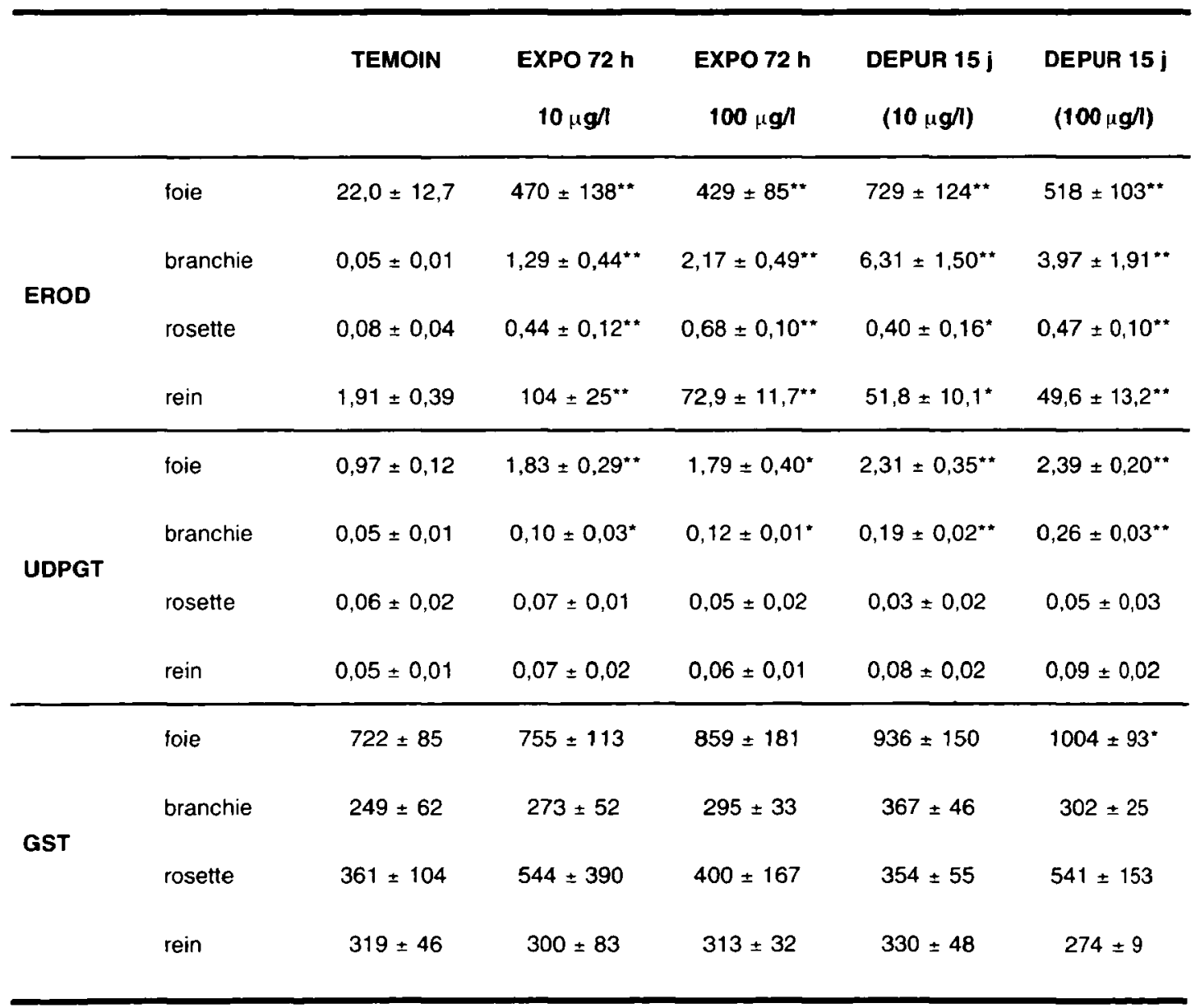




\section{DISCUSSION}

Le $\mathrm{BaP}$ et le TCB sont des inducteurs connus du CYP1A hépatique, en particulier chez le poisson (STEGEMAN et LECH, 1991). GODDARD et al. (1987) obtiennent chez Poeciliopsis monacha et Poecilopsis lucida exposés pendant $24 \mathrm{~h}$ à $0,8-1 \mathrm{mg} / \mathrm{l}$ de BaP des niveaux d'activité hépatique pour l'EROD 3 à 4 fois supérieurs à ceux des poissons témoins. ARINC et SEN (1994) décrivent des augmentations d'activités analogues chez la daurade traitée par injection intrapéritonéale de BaP à la dose de $25 \mathrm{mg} / \mathrm{kg}$. LEMAIRE et al. (1992) dans des conditions expérimentales similaires font état chez le bar d'une augmentation de l'activité EROD de 4 à 14 fois suivant le type d'aliment distribué. Dans notre étude, l'effet du BaP sur l'activité EROD hépatique est encore plus nette ( $x 11$ à 33 fois selon la concentration), suggérant une sensibilité particulière de la truite à ce type d'inducteur. L'augmentation de l'activité EROD provoquée par le TCB dans notre étude est comparable à celle obtenue avec ce composé par injection intrapéritonéale (OTTO et MOON, 1995 ; HUUSKONEN et al., 1996). Nos résultats confirment également la grande sensibilité du rein à l'induction du CYP1A par le TCB mise en évidence par ces auteurs.

L'induction des enzymes dépendants du cytochrome P450 chez le poisson concerne non seulement le foie, mais également les tissus extrahépatiques (SMOLOWITZ et al., 1992). Nous avons pu noter en particulier sur le rein et la branchie que ces inductions pouvaient dépasser celles des enzymes hépatiques. L'augmentation d'activité EROD observée sur les rosettes olfactives est, bien que significative, moins importante que celle obtenue sur les autres tissus et en outre moindre que celle récemment rapportée chez des truites traitées à la $\beta$-naphtoflavone (MONOD et al., 1994). Les réponses que nous avons constatées au niveau des différents tissus sont probablement liées au mode d'exposition, comme le suggèrent VAN VELD et al. (1997) qui ont observé chez des fondules (Fundulus heteroclitus), ayant consommé un aliment contenant $10 \mu \mathrm{g} / \mathrm{g}$ de BaP, une induction majeure du CYP1A dans l'intestin alors que l'induction du CYP1A dans les branchies, l'épithélium cardiaque et le système vasculaire, est prépondérante lors d'une contamination par balnéation dans une eau contenant $10 \mu \mathrm{g} / \mathrm{l}$ de BaP.

Les transférases sont nettement moins sensibles à l'induction que les enzymes qui dépendent du CYP1A. Lors d'injections intrapéritonéales de TCB, OTTO et MOON (1995) et HUUSKONEN et al. (1996), ont vu l'activité UDPGT multipliée par 2 à 6 dans le foie et par 2 à 7 dans le rein. Si nos résultats dans le foie sont comparables à ceux de ces auteurs, nous n'avons, dans nos conditions expérimentales, aucun effet significatif pour ce qui concerne le rein. En revanche, l'augmentation importante de l'activité UDPGT branchiale que nous avons observée n'a à notre connaissance jamais été décrite.

La différence majeure entre le traitement au BaP et celui au TCB concerne les valeurs de l'activité EROD à la fin de la période de dépuration. Dans le premier cas, les activités enzymatiques reviennent à leur niveau initial lorsque les poissons sont placés en milieu non contaminé, alors que l'augmentation d'activité se poursuit au cours de la période de dépuration chez les truites exposées au TCB. Ce phénomène est vraisemblablement dû à l'élimination rapide du BaP par rapport au TCB chez le poisson. SANDVIK et al. (1997) ont montré que la demi-vie du BaP chez des truites exposées à cet hydrocarbure dans l'eau variait de 2,9 jours dans le foie à 6,2 jours dans les branchies. Ces valeurs sont supérieures à 100 jours pour le TCB (NIIMI et OLIVER, 1983).

\section{CONCLUSION}

Nos travaux confirment la grande sensibilité de l'activité EROD des truites à des contaminants tels que les HAP ou les PCB. En regard de l'utilisation de cette activité comme biomarqueur de pollution, nos résultats montrent qu'une exposition transitoire à des 
hydrocarbures aromatiques polycycliques tels que le BaP ne pourra pas être repérée si les prélèvements interviennent tardivement ( 15 jours ou plus après la contamination). La situation est clairement différente pour le TCB pour lequel l'induction se poursuit bien audelà de la période de contact entre le polluant et le poisson. Pour ces xénobiotiques, il serait opportun de poursuivre l'expérimentation en prolongeant la période de dépuration de plusieurs semaines afin d'évaluer la durée de la réponse enzymatique aux PCB.

\section{REMERCIEMENTS}

Les auteurs tiennent à remercier N. DELLANDES pour son excellente collaboration technique.

\section{BIBLIOGRAPHIE}

ANDERSSON T., PESONEN M., JOHANSSON C., 1985. Differential induction of cytochrome P-450-dependent monooxygenase, epoxide hydrolase, glutathione transferase and UDP glucuronosyl transferase activities in the liver of rainbow trout by $\beta$-naphthoflavone or Clophen A50. Biochem. Pharmacol., 34, 3309-3314.

ARINC E., SEN A., 1994. Effects of in vivo benzo(a)pyrene treatment on liver microsomal mixed-function oxidase activities of gilt head sea bream (Sparus aurata). Comp. Biochem. Physiol., 107C, 405-414.

BOULOUBASSI I., SALIOT A., 1993. Aquatic distribution and origin of PAH in coastal deltaic waters. Polycyclic Aromatic Compounds, 3, 379-388.

CHEVREUIL M., GRANIER L., CARRU A.M., 1995. Relationship between biological parameters and bioaccumulation of some organochlorines (pesticides, PCB) by fishes in the river Seine (France). Water, Air and Soil Pollution, 81, 107-120.

GODDARD K.A., SCHULTZ R.J., STEGEMAN J.J., 1987. Uptake, toxicity and distribution of benzo(a)pyrene and monooxygenase induction in the topminnows Poeciliopsis monacha and Poeciliopsis lucida. Drug Metab. Dispos., 15, 449-455.

GOKSøYR A., FÖRLIN L., 1992. The cytochrome P-450 system in fish, aquatic toxicology and environmental monitoring. Aquat. Toxicol., 22, 287-312.

HAASCH M.L., PRINCE R., WEJKSNORA P.J., COOPER K.R., LECH J.J., 1993. Caged and wild fish : Induction of hepatic cytochrome P450 (CYP1A1) as an environmental biomonitor. Environ. Toxicol. Chem., 12, 885-895.

HABIG W.H., JAKOBY W.B., 1981. Assays for differenciation of glutathione S-transferases. Meth. Enzymol., 77, 398-405.

HUUSKONEN S., LINDSTRÖM-SEPPÄ P., KOPONEN K., ROY S., 1996. Effects of nonortho-substituted polychlorinated biphenyls (congeners 77 and 126) on cytochrome $\mathrm{P} 4501 \mathrm{~A}$ and conjugation activities in rainbow trout (Oncorhynchus mykiss). Comp. Biochem. Physiol., 113C, 205-213.

JEDAMSKI-GRYMLAS J., KAMMANN U., TEMPELMAN A., KARBE L., SIEBERS D., 1995. Biochemical responses and environmental contaminants in breams (Abramis brama L.) caught in the river Elbe. Ecotoxicol. Environ. Sat., 31, 49-56.

JONES J.G., HORNE J.E.M., MOORHOUSE P., POWELL D.L., 1980. Petroleum hydrocarbons in fresh waters. Freshwater Biological Association Occasional Publication, $\mathrm{N}^{\circ} 9,52 \mathrm{p}$.

LEMAIRE P., MATHIEU A., GIUDICELLI J., LAFAURIE M., 1992. Effect of diet on the responses of hepatic biotransformation enzymes to benzo(a)pyrene in the European sea bass (Dicentrarchus labrax). Comp. Biochem. Physiol., 102C, 413-420. 
LINDSTRÖM-SEPPÄ P., KOIVUSAARI U., HÄNNINEN O., PYYSALO H., 1985. Cytochrome P-450 and monooxygenase activities in the biomonitoring of aquatic environment. Pharmazie, 40, 232-234.

LOWRY O.H., ROSEBROUGH N.J., FARR A.L., RANDALL R.J., 1951. Protein measurements with the Folin phenol reagent. J. Biol. Chem., 193, 265-275.

MONOD G., DEVAUX A., RIVIERE J.L., 1988. Effects of chemical pollution on the activities of hepatic xenobiotic metabolizing enzymes in fish from the river Rhône. Sci. Tot. Environ., 73, 189-201.

MONOD G., SAUCIER D., PERDU-DURAND E., DIALLO M., CRAVEDI J.P., ASTIC L., 1994. Biotransformation enzyme activities in the olfactory organ of rainbow trout (Oncorhynchus mykiss). Immunocytochemical localization of cytochrome P4501A1 and its induction by $\beta$-naphthoflavone. Fish Physiol. Biochem., 13, 433-444.

NARBONNE J.F., GARRIGUES P., RIBERA D., RAOUX C., MATHIEU A., LEMAIRE P., SALAUN J.P., LAFAURIE M., 1991. Mixed-function oxygenase enzymes as tools for pollution monitoring : field studies on the French coast of the Mediterranean sea. Comp. Biochem. Physiol., 100C, 37-42.

NIIMI A.J., OLIVER B.G., 1983. Biological half-lives of polychlorinated biphenyl (PCB) congeners in whole fish and muscle of rainbow trout (Salmo gairdneri). Can. J. Fish. Aquat. Sci., 40, 1388-1394.

OTTO D.M.E., MOON T.W., 1995. 3,3', 4,4'-tetrachlorobiphenyl effects on antioxidant enzymes and glutathione status in different tissues of rainbow trout. Pharmacol. Toxicol., 77, 281-287.

PAYNE J.F., FANCEY L.L., RAHIMTULA A.D., PORTER E.L., 1987. Review and perspective on the use of mixed-function oxygenase enzymes in biological monitoring. Comp. Biochem. Physiol., 86C, 233-245.

SANDVIK M., HORSBERG T.E., SKAARE J.U., INGEBRIGTSEN K., 1997. Hepatic CYP1A induction in rainbow trout (Oncorhynchus mykiss) after exposure to benzo(a)pyrene in water. Biomarkers, 2, 175-180.

SMOLOWITZ R.M., SCHULTZ M.E., STEGEMAN J.J., 1992. Cytochrome P4501A induction in tissues, including olfactory epithelium, of topminnows (Poeciliopsis spp.) by waterborne benzo(a)pyrene. Carcinogenesis, 13, 2395-2402.

STEGEMAN J.J., LECH J.J., 1991. Cytochrome P-450 monooxygenase systems in aquatic species : carcinogen metabolism and biomarkers for carcinogen and pollutant exposure. Environ. Health Pespect., 90, 101-109.

TUKEY R.H., BILLINGS R.E., TEPHLY T.H., 1978. Separation of oestrone UDPglucuronyltransferase and p-nitrophenol UDP-glucuronyltransferase activities. Biochem. J., 171, 659-663.

VAN VELD P., VOGELBEIN W.K., COCHRAN M.K., GOKSØYR A., STEGEMAN J.J., 1997. Route-specific cellular expression of cytochrome P4501A (CYP1A) in fish (Fundulus heteroclitus) following exposure to aqueous and dietary benzo(a)pyrene. Toxicol. Applied Pharmacol., 142, 348-359.

VINDIMIAN E., GARRIC J., 1989. Freshwater fish cytochrome P450-dependent enzymatic activities : a chemical pollution indicator. Ecotoxicol. Environ. Saf., 18, 277-285. 